\title{
An Inquiry into the Management Theory: Introducing Cooperative Advantage
}

\author{
Murat Kayak ${ }^{1}$ \\ Ph.D. in Management, XXX Cycle \\ Department of Economics Management, Institutions \\ University of Naples Federico II \\ Naples, Italy
}

The full paper is available at Altmetric - https://cambridge.altmetric.com/details/83747834

Citations:

APA

Kayak, M. (2020, June 9). An Inquiry into the Management Theory: Introducing Cooperative Advantage. Cambridge Open Engage. https://doi.org/10.33774/coe-2020-spzvh

MLA

Kayak, Murat. “An Inquiry into the Management Theory: Introducing Cooperative Advantage.” Cambridge Open Engage., 9 Jun. 2020. Web.

\section{Chicago}

Kayak, Murat. 2020. “An Inquiry into the Management Theory: Introducing Cooperative Advantage.” Cambridge Open Engage. June 9. doi:10.33774/coe-2020-spzvh.

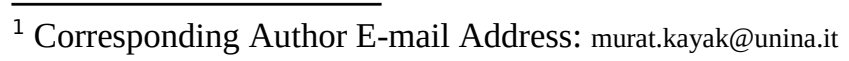




\begin{abstract}
Although scientists and philosophers have debated epistemological questions since the time of Plato and Aristotle, the term Epistemology is not usually noticed in management research as mentioned in the literature by Johnson and Duberley in 2000. However, epistemology is still not usually noticed in management research. The most commonly known epistemological approaches are interpretative and positivism. The purpose of this study is to introduce a cooperative advantage model in management by addressing epistemology. The study focuses on how competitive advantage damage humankind. The study also figures out whether Management is Science or Not. The study findings show that management in fact is science and philosophy however scholars of the 20th and 21st century have made management just a discipline based on the puzzle-solving approach introduced by Thomas Kuhn in 1962. I sincerely hope the scientific community to pay attention to this research.
\end{abstract}

\title{
(C) 2020 Murat Kayak
}

Keywords: Management, Cooperative Advantage, Theory

Biography: Murat Kayak awarded his Ph.D. from the University of Naples Federico II (Naples - Italy), Master from Asia University (Taichung - Taiwan), and Bachelor from Mugla University (Muğla Türkiye). 